\title{
White Dwarf Mass Estimation with X-ray Spectroscopy
}

\section{Takayuki Hayashi*}

Department of Physics, University of Maryland Baltimore County, 1000 Hilltop Circle, Baltimore, Maryland 21250, USA

NASA, Goddard Space Flight Center, Code 662, Greenbelt, MD20771, USA

E-mail: takayuki.hayashienasa.gov

X-ray thermal and reflection spectral models of intermediate polars (IPs) are introduced. The thermal model is based on a hydrodynamical calculation for the Post-Shock Accretion Column (PSAC). The total PSAC spectrum is obtained by integrating the spectrum along the PSAC with the calculated plasma parameters. The thermal spectrum is harder with a more massive WD because of its deeper potential. The reflection is modeled by a Monte-Carlo simulation. The PSAC irradiates the cool WD with the various thermal X-ray spectra by considering the stratified structure of the PSAC. Basically, the reflection spectrum is harder and more intense relative to the thermal with a more massive WD. The IP spectral models were applied to V1223 Sagittarii and reasonably reproduce its spectrum. The WD mass and accretion rate per unit area (specific accretion rate) were measured to be $M_{\mathrm{WD}}=0.92 \pm 0.02 M_{\odot}$ and $a>10^{0.3} \mathrm{~g} \mathrm{~cm}^{-2} \mathrm{~s}^{-1}$, respectively.

Frontier Research in Astrophysics - III (FRAPWS2018)

28 May - 2 June 2018

Mondello (Palermo), Italy

\footnotetext{
*Speaker.
} 


\section{Introduction}

Cataclysmic variables (CVs) are binary systems of a white dwarf (WD) and a Roche Lobefilling late type star. CVs whose WD is strongly magnetized $\left(B_{\mathrm{WD}}>0.1 \mathrm{MG}\right)$ are called magnetic CVs (mCVs). The mCVs with $B_{\mathrm{WD}}>10 \mathrm{MG}$ and $0.1<B_{\mathrm{WD}}<10 \mathrm{MG}$ are classified into mCV subclasses of polars and intermediate polars (IPs), respectively. In the IPs, the accretion disc forms around the WD but it is truncated by the WD magnetic field at around the Alfvén radius. By contrast, in polars, the disc is not formed and the magnetic field locks the companion star. The accreting matter caught by the magnetic field falls along the magnetic field at almost free fall velocity. The accreting flow forms a strong shock near the WD surface and the accreting matter is heated up to $\sim 10^{8} \mathrm{~K}$ and highly ionized. The hot plasma cools down as it approaches the WD surface. This cooling plasma flow is called a Post-Shock Accretion Column (PSAC).

The temperature of the PSAC depends on the WD gravitational potential. Thus, the WD mass can be measured by measuring the plasma temperature with X-ray spectroscopy with the aid of theoretical WD mass-radius relations (e.g. [ए]]). In modern technique, multi-temperaure

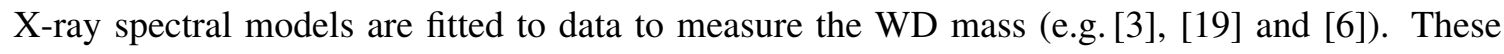
$\mathrm{X}$-ray spectral models rely on hydrodynamical calculations and include the stratified structure of the PASC.

Recently, X-ray spectrum of reflection from the WD, which is the other major X-ray source in the mCVs, has been modeled in detail [ $[\mathrm{C}]$. The reflection component is X-rays which are originally emitted from the PSAC and reprocessed on the WD surface. The reprocessing is due to the scattering and the reemission accompanying the photoelectric absorption by the atoms. The reflection component makes mainly four features in its spectrum; (1) the iron K-shell lines (2) the iron K-edge (3) the Compton shoulder below emission lines and (4) the Compton hump at 20-30 keV. The detailed reflection model reduces the mixture of the thermal and the reflection spectra. Moreover, the intensity of the reflection depends on the solid angle of the WD surface viewing from the plasma. The solid angle gives us the height of the plasma from the WD surface, which is important information to determine the WD mass. As a result, the accuracy of the mass measurement is enhanced by the reflection model.

In $\$$, I introduce PASC thermal and the reflection models of the IPs. Their application to a typical IP V1223 Sgr is shown in $\S[$ as a example. Finally, I summarize the models and their application in $\S$.

\section{Spectral model}

\subsection{Thermal emission}

I introduce here a thermal X-ray spectral model for the IPs proposed by [目]. This model is based on the model of [B] and includes several physical effects.

The mass continuity equation of steady quasi-one-dimensional flows is

$$
\frac{\mathrm{d}}{\mathrm{d} z}(\rho v S)=0
$$

where $z$ is the spatial coordinate along the flow, $\rho$ is the mass density, $v$ is the velocity of the bulk motion and $S$ is the cross section of the flow. Here, the WD center is chosen as the origin of $z$. The 
integral form of the equation 2 .

$$
\rho v S=a S_{0}=\text { const }
$$

where $\rho v$ is the mass flux (in $\mathrm{g} \mathrm{cm}^{-2} \mathrm{~s}^{-1}$ ) and, especially, $a$ is the accretion rate per unit area and $S_{0}$ is the flow cross-section at the WD surface in our case. $a$ is called specific accretion rate, which is one of the most important parameter as well as the WD mass. The one dimensional momentum and energy equations are written as

$$
\frac{\mathrm{d}}{\mathrm{d} z}\left(\rho v^{2}+P\right)=-\frac{G M_{\mathrm{WD}}}{z^{2}} \rho-\frac{\rho v^{2}}{S} \frac{\mathrm{d} S}{\mathrm{~d} z}
$$

and

$$
v \frac{\mathrm{d} P}{\mathrm{~d} z}+\gamma P \frac{\mathrm{d} v}{\mathrm{~d} z}=-(\gamma-1)\left(\varepsilon-\frac{\rho v^{3}}{2 S} \frac{\mathrm{d} S}{\mathrm{~d} z}\right)
$$

respectively, with the use of the equation 2.2. $T$ is the averaged temperature, $P$ is the thermal pressure and $\gamma=5 / 3$ is the adiabatic index. $\varepsilon$ is the cooling rate via radiation. Here, the plasma assumed to be optically thin and, therefore, $\varepsilon$ is given by

$$
\varepsilon=\left(\frac{\rho}{\mu_{\mathrm{n}} m_{\mathrm{H}}}\right)^{2} \Lambda(T)
$$

where $\mu_{\mathrm{n}}=1.2$ is the mean molecular weight of a neutral gas of the solar abundance and $\Lambda$ is the cooling function. In our case, the Collisional Ionization Equilibrium (CIE) cooling function calculated by [[1]] is used. This cooling function includes the line emission as well as the bremsstrahlung. I note that we have to include the cyclotron cooling into the cooling function for the polars but do not have to do so for the IPs ([R] ). The terms of $\frac{d S}{d z}$ in the equations of 2.3 and $[2.4$ represent acceleration of the flow and energy conversion in the flow due to the nozzle effect, respectively. In this model, a shape of the flow is assumed to be the dipolar for the PSAC, and, therefore, $S$ is proportional to $z^{3}$.

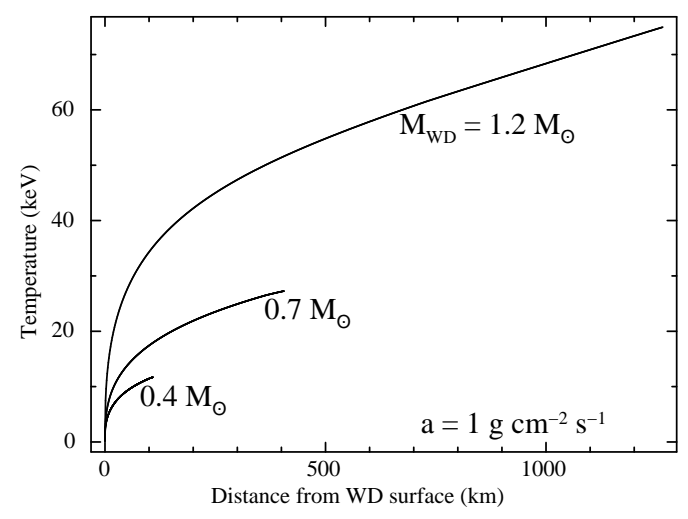

Figure 1: Temperature distributions of the PSAC with the WD mass of $M_{\mathrm{WD}}=0.4,0.7$ and $1.2 M_{\odot}$.

The flow of the PSAC can be calculated with the equations $[2.3$ and 2.4 with the ideal-gas law

$$
P=\frac{\rho k T}{\mu_{\mathrm{i}} m_{\mathrm{H}}}
$$


where $\mu_{\mathrm{i}}=0.62$ is the mean molecular weight of a fully ionized plasma of the solar abundance. The strong shock approximation at the shock and soft landing ( $v=0$ at the WD surface) are assumed as the initial condition and the boundary condition, respectively. Moreover, the WD mass-radius

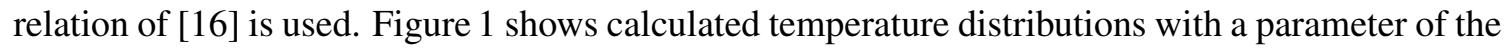
WD mass. More massive WDs makes a hotter PSAC because of their deeper potential. The hotter plasma needs longer cooling time and, therefore, makes the PSAC taller.

The thermal PSAC X-ray spectral model can be obtained with integrating the X-ray spectra along the PSAC. Concretely, the distributions of physical quantities are divided into logarithmically spaced 100 segments. The spectra of the segments are calculated by SPEX package [ए0] by inputting the calculated physical quantities in each segment. The whole spectrum was obtained by summing the 100 spectra.

Figure $\square$ shows the constructed thermal spectra (dotted lines) with the WD mass of $M_{\mathrm{WD}}=0.4$ (black) and 1.2 (green) $M_{\odot}$. A more massive WD makes harder spectrum with its deeper potential.
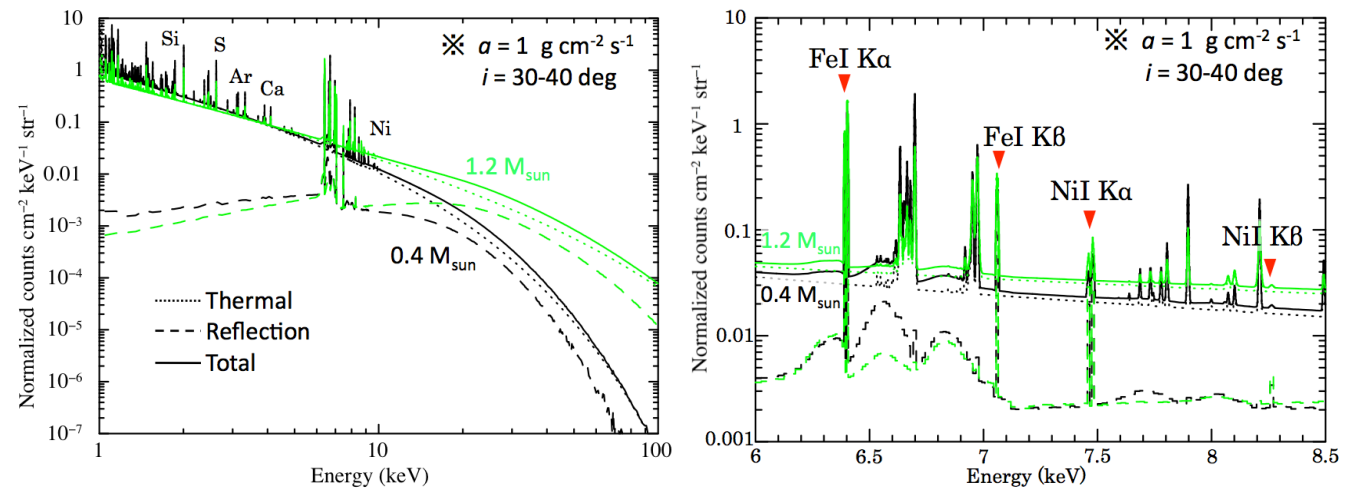

Figure 2: Model spectra with the WD mass of $M_{\mathrm{WD}}=0.4$ (black) and 1.2 (green) $M_{\odot}$. Dotted, dashed and solid lines show the spectra of the thermal, reflection and the sum of them.

\subsection{Reflection}

In general, the reflection is modeled by Monte-Carlo simulation. In the modeling by [ $[\mathrm{D}]$, the PSAC irradiates the WD with the various thermal X-ray spectra from the corresponding heights according to the stratified PSAC structure ( $\$[$. The cool WD means that the atoms in the WD is neutral and X-rays interact with the atoms by the scattering and the photoelectric absorption. The photoelectric absorption is accompanied by the reemission with the fluorescence yield. Moreover, there two kinds of scattering, the coherent scattering and the incoherent scattering unlike the scattering by the free electrons (Compton scattering). In this simulation, the coherent and the incoherent scatterings, the photoelectric absorption and the reemission of $\mathrm{K} \alpha$ and $\mathrm{K} \beta$ of $\mathrm{Fe}$ and $\mathrm{Ni}$ were taken into account. A photon continued to interact until that photon escapes from the WD until its energy goes down below $1 \mathrm{keV}$ or completely absorbed.

Mass attenuation coefficients (figure[3) by the NIST (National Institute of Standards and Technology) based on [9] were used in this simulation. The photoelectric absorption is the most significant process up to $\sim 10 \mathrm{keV}$. Above the $10 \mathrm{keV}$, the incoherent scattering is the prime process. 
Although the coherent scattering is a minor process at any energy, this process is significant for forward scattering (see below).

The energies of the $\mathrm{K}$-edges of $\mathrm{Fe}$ and $\mathrm{Ni}$, whose fluorescent $\mathrm{K} \alpha$ and $\mathrm{K} \beta$ lines are included in the simulation, are assumed to be $7.11 \mathrm{keV}$ and $8.33 \mathrm{keV}$, respectively. The reemission can occur when an X-ray whose energy is higher than the edge energies absorbed by the K-shell of the Fe or $\mathrm{Ni}$ atom. The K-shell fluorescence yields of $\mathrm{Fe}$ and $\mathrm{Ni}$ are 0.34 and 0.38 , respectively, which determine the possibility of the $\mathrm{K}$ line reemission for the stimulated atom. The line ratios of $\mathrm{K} \alpha$ and $\mathrm{K} \beta$ of $\mathrm{Fe}$ and $\mathrm{Ni}$ are assumed to be 150:17 and 151:17 [प]], respectively.

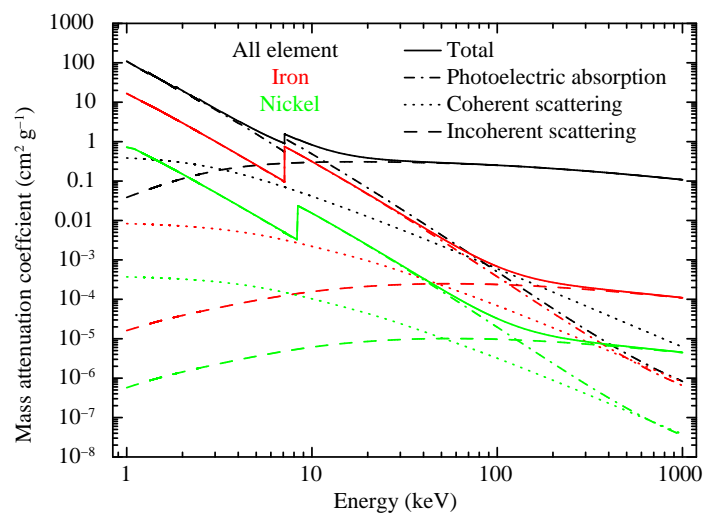

Figure 3: Mass attenuation coefficient of one solar abundance (adopted from [四]). The dashed-dotted, dotted, dashed curves are the photoelectric absorption, the coherent and incoherent scatterings, respectively. Solid line is the total of them. Black lines represent the mass attenuation coefficient of the all element, while red and green lines show fractional ones of iron and nickel, respectively.

Figure田 shows the differential cross sections of the coherent and incoherent scatterings. Low energy X-rays such as $6.4 \mathrm{keV}$ have the non-negligible differential cross section in every direction. Especially for the forward scattering with the scattering angle $\lesssim 30 \mathrm{deg}$, the coherent scattering is important. Higher energy X-rays such as $50 \mathrm{keV}$ have the negligible differential cross section for the backward scattering with the scattering angle $\gtrsim 90 \mathrm{deg}$. Even with the higher energy, the forward scattering with the small scattering angle $\lesssim 5 \mathrm{deg}$ is significant.

Energy of the X-rays scattered by the incoherent scattering is affected by the ionization energy and the momentum of the scattering electron bound in the atom because of the Doppler effect (Compton profile) differently from the Compton scattering. To calculate the energy, complicated calculation is needed, which is omitted here (see [ [ $]$ ] for deital).

The dashed lines in the figure $\square$ show the simulated reflection X-ray spectra. The X-rays irradiated or processed inside of the WD go through the WD atmosphere and prominent edges are made (The optical depth the X-rays go through is larger when the reflected X-rays go to a larger reflecting angle from the PSAC (i) and the reflection is weaker. See [ $[$ ] for deital). Consequently, the fluorescent of $\mathrm{Fe}-\mathrm{K} \alpha, \beta$ and $\mathrm{Ni}-\mathrm{K} \alpha, \beta$ lines are formed, which are accompanied by their red tail due to the incoherent scattering (Compton shoulder). Thermal emission lines make their Compton shoulder in the reflection spectra as well. Moreover, there is a hump (Compton hump) between 20 and $30 \mathrm{keV}$ where the prime process changes from the incoherent scattering to the photoelectric absorption for the X-rays whose energy is decreasing. In most cases, the reflection is harder and 
more intense relative to the thermal with a more massive WD because the irradiating thermal spectrum is harder and difficult to be absorbed in the WD atmosphere. On the other hand, the PSAC is taller with a more massive WD (figureW), which weakens the reflection. Thus, in some cases, the reflection is weaker with the more massive WD. The solid lines in the figure $\square$ are the sum of the thermal and reflection components to be applied to the observation.

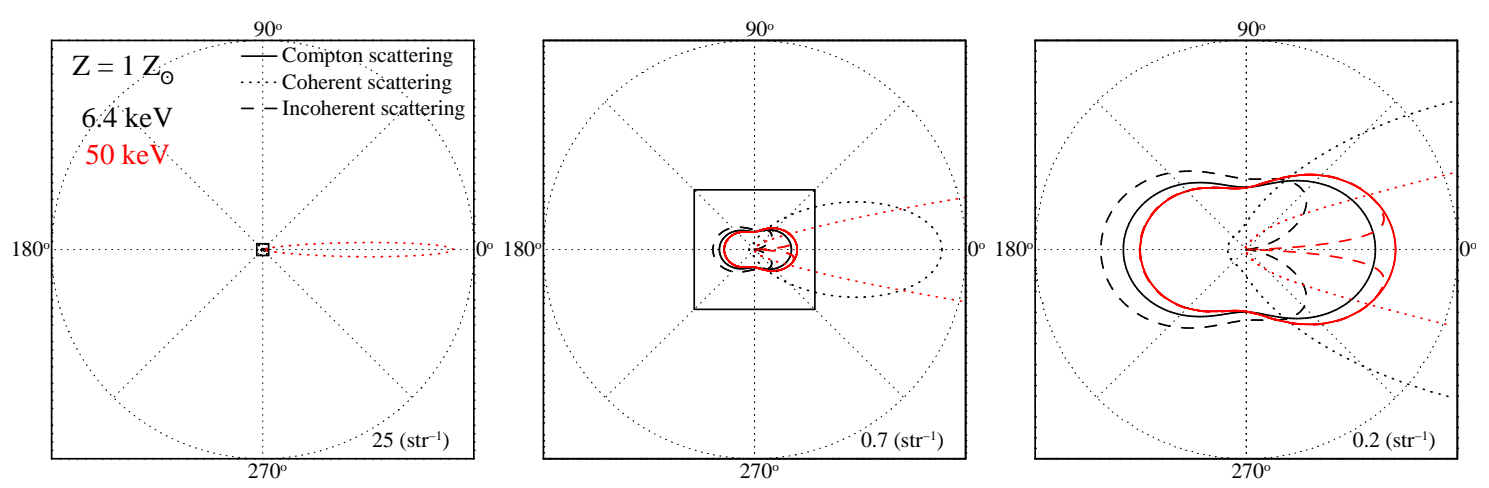

Figure 4: Differential cross sections for an incident X-ray of $6.4 \mathrm{keV}$ (black) and $50 \mathrm{keV}$ (red) scattered off by one solar abundance material (adopted from [ $[\mathbf{]}]$ ). Solid, dotted and dashed lines show the differential cross sections of Compton scattering, coherent scattering and incoherent scattering by bound electrons, respectively. These differential cross sections are normalized so that each total cross section becomes unity. The middle panel is a blowup of the central square in the left panel. The right panel is again a blowup of the central square in the middle panel.

\section{Model application}

The introduced spectral models were applied to one of the brightest IP 1223 Sagittarii (see [8] for detail). The archival data of the Suzaku satellite [14]] and NuSTAR satellite [[]] were used. Suzaku observed V1223 Sgr in April 2007 and, March and April 2014. The NuSTAR observed it in September 2014.

Suzaku has three front-illuminated CCDs (XIS0, 2 and 3; note that XIS2 is unusable at these observations in 2007) and one back-illuminated CCD (XIS1) [[3]. Their energy resolutions are 150-160 eV. Each XIS is located at focus of the dedicated X-ray telescope (XRT0-3; [18]). Each of XRTs has effective area of $440 \mathrm{~cm}^{2}$ at $1.5 \mathrm{keV}$ and $250 \mathrm{~cm}^{2}$ at $8 \mathrm{keV}$. The hard X-ray above $10 \mathrm{keV}$ is covered by a non-imaging, collimated detector (HXD; [20] and [ए2]). It has two components. One of them is a PIN detector sensitive to X-rays of $10-70 \mathrm{keV}$ and the other is a GSO/BGO phoswitch counter sensitive to 40-600 keV. Here, only PIN was used because GSO/BGO detected no significant signal. The exposures time of XIS and HXD are $240.9 \mathrm{ksec}$ and $218.7 \mathrm{ksec}$ in total, respectively.

NuSTAR has two co-aligned hard X-ray telescopes which focus hard X-rays $(3-79 \mathrm{keV})$ [[]]. Each telescope focuses celestial X-rays on a Focal Plane Module (FPM), which is called FPMA or FPMB. Effective area of the FPMAs is $100 \mathrm{~cm}^{2}$ at $50 \mathrm{keV}$ [] $]$ and its energy resolution is $0.4 \mathrm{keV}$ below $50 \mathrm{keV}$ and rises to $1.0 \mathrm{keV}$ at $86 \mathrm{keV}$ [ए5]]. The exposure time of the FPMs is $20.4 \mathrm{ksec}$.

Figure $\square$ shows the model application to V1223 Sgr with the photoelectric absorption attenuation. In this application, the spectrum above $5 \mathrm{keV}$ was fitted to avoid a complicated multicolumn 


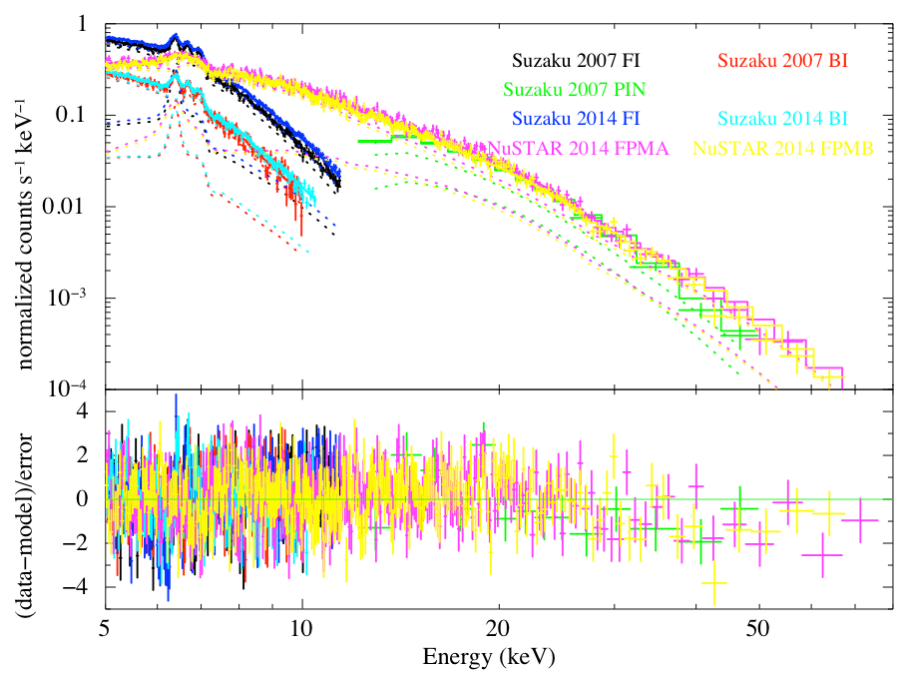

Figure 5: Suzaku-2007 FI (black), Suzaku-2007 BI (red), Suzaku-2007 PIN (green), Suzaku-2014 FI (blue), Suzaku-2014 BI (light blue), NuSTAR-2014 FPMA (magenta) and NuSTAR- 2014 FPMB (yellow) spectra with the best-fit of the IP model attenuated by the photoelectric absorption. The lower panel shows the residual in units of $\sigma$.

absorption [ [9]. The V1223 Sgr X-ray spectrum is reasonably reproduced by the models and its reduced- $\chi^{2}$ and d.o.f. are 1.33 and 1192, respectively. The WD mass of V1223 Sgr and the specific accretion rate were measured to be $M_{\mathrm{WD}}=0.92 \pm 0.02 M_{\odot}$ and $a>10^{0.3} \mathrm{~g} \mathrm{~cm}^{-2} \mathrm{~s}^{-1}$, respectively.

\section{Summary}

In this paper, I introduce the IP spectral model including the thermal and the reflection components. The thermal model is based on a quasi-one-demenstional hydrodynamical calculation for the PSAC. The total PSAC spectrum is obtained by integrating the spectrum along the PSAC which is acquired by an existing plasma emission model by SPEX with the calculated plasma parameters. The thermal spectrum is harder with a more massive WD because of its deeper potential.

The reflection is modeled by a Monte-Carlo simulation with the hydrodynamical and the thermal spectral models. The PSAC irradiates the cool and neutral WD with the various thermal X-ray spectra from the corresponding heights according to the hydrodynamical calculation. The simulated spectrum includes the strong $\mathrm{Fe}$ edge, the fluorescent $\mathrm{K} \alpha, \beta$ lines of $\mathrm{Fe}$ and $\mathrm{Ni}$, the Compton shoulder of lines and the Compton hump. Basically, the reflection spectrum is harder and more intense relative to the thermal with a more massive WD.

The IP model was applied to V1223 Sgr observations of Suzaku and NuSTAR satellites. The model reasonably reproduces the V1223 Sgr X-ray spectrum. The WD mass and the specific accretion rate were measured to be $M_{\mathrm{WD}}=0.92 \pm 0.02 M_{\odot}$ and $a>10^{0.3} \mathrm{~g} \mathrm{~cm}^{-2} \mathrm{~s}^{-1}$, respectively.

The plasma parameters in the IPs are still controversial. Especially, the density is difficult to be measured. XRISM (X-Ray Imaging and Spectroscopy Mission) satellite will be launched in 2022. It is going to resolve the triplet of the He-like Fe-K $\alpha$ lines (resonance, forbidden and intercombination). The critical density of the He-like Fe is $10^{17-18} \mathrm{~cm}^{-3}$ which presumably agrees 
with the density of the IP plasma. The line ratio of the forbidden to the intercombination directly gives us the density and refine the IP hydrodynamical model and, therefore, the spectral models.

\section{References}

[1] Aizu, K. 1973, Progress of Theoretical Physics, 49, 1184

[2] Craig W. W., et al., 2011, SPIE, 8147, 81470H

[3] Cropper, M., Wu, K., Ramsay, G., \& Kocabiyik, A. 1999, MNRAS, 306, 684

[4] Ezuka H., Ishida M., 1999, ApJS, 120, 277

[5] Harrison F. A., et al., 2013, ApJ, 770, 103

[6] Hayashi, T., \& Ishida, M. 2014, MNRAS, 438, 2267

[7] Hayashi T., Kitaguchi T., Ishida M., 2018, MNRAS, 474, 1810

[8] Hayashi T., Kitaguchi T., Ishida M., MNRAS, in prep

[9] Hubbell J. H., Veigele W. J., Briggs E. A., Brown R. T., Cromer D. T., Howerton R. J., 1975, JPCRD, 4,471

[10] Kaastra, J. S., Mewe, R., \& Nieuwenhuijzen, H. 1996, UV and X-ray Spectroscopy of Astrophysical and Laboratory Plasmas, 411

[11] Kikoin, I. K. 1976, Tables of Physical Quantities (Moscow: Atomizdat)

[12] Kokubun, M., Makishima, K., Takahashi, T., et al. 2007, PASJ, 59, 53

[13] Toyama, K., Tsunami, H., Dotani, T., et al. 2007, PASJ, 59, 23

[14] Mitsuda, K., Bautz, M., Inoue, H., et al. 2007, PASJ, 59, 1

[15] Rana V. R., Cook W. R., III, Harrison F. A., Mao P. H., Miyasaka H., 2009, SPIE, 7435, 743503

[16] Nauenberg, M. 1972, ApJ, 175, 417

[17] Schure, K. M., Kosenko, D., Kaastra, J. S., Keppens, R., \& Vink, J. 2009, A\&A, 508, 751

[18] Serlemitsos, P. J., Soong, Y., Chan, K.-W., et al. 2007, PASJ, 59, 9

[19] Suleimanov V., Revnivtsev M., Ritter H., 2005, A\&A, 435, 191

[20] Takahashi, T., Abe, K., Endo, M., et al. 2007, PASJ, 59, 35

[21] Wu K., Chanmugam G., Shaviv G., 1994, ApJ, 426, 664

\section{DISCUSSION}

DMITRY BISIKALO: From past observations and simulations, we know that the shape of the "hot spot" is not a spot but a curtain. It seems that it can change your results significantly.

TAKAYUKI HAYASHI: That may be true. I should include more realistic shape for the accretion column. 
WOLFGANG KUNDT: How certain are you that the white dwarf's magnetsphere has of an axisparallel dipole?

TAKAYUKI HAYASHI: Here, I just assumed the dipole. I should include more realistic shape.

WOLFGANG KUNDT: Can a toroidally strangled multipole structure be excluded?

TAKAYUKI HAYASHI: The X-ray spin modulation is sinusoidal. So, multipole is not likely.

Gennady BISNOVATYI-KOGAN: How do you find the distance between the shock and WD surface.

TAKAYUKI HAYASHI: The shock height depends on the WD mass the specific accretion rate and so on. They are included into my model as the fitting parameters. So, the shock height is adjusted to reproduce the spectrum via the fitting parameters. 